where the large letters are the cofactors of the corresponding small letters in $\Delta_{n}$. It will be noticed that each member of (6) is of degree $3 n$, as it should be.

UNIVERSITY OF WASHINGTON, March, 1911.

\title{
NOTE ON THE MAXIMAL CYCLIC SUBGROUPS OF A GROUP OF ORDER $p^{m}$.
}

BY PROFESSOR G. A. MILLER.

(Read before the San Francisco Section of the American Mathematical Society, October 28, 1911.)

IF $H$ is any non-invariant subgroup of a group $G$ of order $p^{m}$, $p$ being any prime number, it is well known that $H$ is transformed into itself by at least one of its conjugates under $G$ and hence by operators which are not contained in $H .^{*}$ If $H$ is cyclic and not contained in a larger cyclic subgroup of $G$, it is said to be a maximal cyclic subgroup of $G$. In what follows we shall establish the

TheOREM: A necessary and sufficient condition that every maximal cyclic subgroup of order $p^{\alpha}$ in a group $G$ of order $p^{m}, m>3$, is transformed into itself by no more than $p^{a+1}$ operators of $G$ is that $G$ contains one and only one cyclic subgroup of order $p^{m-1}$.

If we combine with this theorem some well-known properties of the groups of order $p^{m}$ which contain operators of order $p^{m-1}$, it results that there are only three non-cyclic groups of order $p^{m}$ which have the property that each of their maximal cyclic subgroups of order $p^{\alpha}$ is transformed into itself by only $p^{a+1}$ operators of the group. These three groups are the three non-cyclic groups of order $2^{m}$ which involve one and only one cyclic subgroup of order $2^{m-1}$.

To prove the theorem in question, we shall assume that $G$ does not involve any operator of order $p^{m-1}$, since the groups of order $p^{m}$ which contain operators of order $p^{m-1}$ are so well known. We shall also assume in what follows that $G$ satisfies the condition that each one of its maximal cyclic subgroups of order $p^{a}$ is transformed into itself by exactly $p^{a+1}$ operators of $G, p^{a}$ being the order of any one of the maximal cyclic subgroup of $G$.

* Cf. American Journal of Mathematics, vol. 23 (1901), p. 173. 
If $H$ is a maximal cyclic subgroup of order $p^{a}$ contained in $G$, it must have $p^{m} / p^{a+1}$ conjugates under $G$ and these conjugates must involve

$$
\frac{p^{m}}{p^{\alpha+1}}\left(p^{\alpha}-p^{\alpha-1}\right)=p^{m}\left(\frac{1}{p}-\frac{1}{p^{2}}\right)
$$

distinct operators of order $p^{a}$. Since any complete set of conjugates of a non-cyclic group of order $p^{m}$ is contained in a subgroup of order $p^{m-1}$, it results that these $p^{m}\left(\frac{1}{p}-\frac{1}{p^{2}}\right)$ operators of order $p^{\alpha}$ in $G$ must generate a subgroup of order $p^{m-1}$ This subgroup includes a subgroup $K$ of order $p^{m-2}$ which does not involve any generator of the cyclic subgroups which are conjugate with $H$, but it is composed of all the other operators of the given subgroup of order $p^{m-1}$. Since $G$ involves $p^{m}-p^{m-1}$ operators which are generators of maximal cyclic subgroups but are not found in the given subgroup of order $p^{m}$, it results that $G$ contains exactly $p+1$ distinct conjugate sets of maximal cyclic subgroups.

As each of these distinct sets generates a subgroup of order $p^{m-1}$ which involves $K$, it results that $K$ is a characteristic subgroup of $G$ which gives rise to an abelian quotient group of order $p^{2}$ and of type $(1,1)$. As all the operators of $K$ are powers of larger operators which are not in $K$, it results that $K$ cannot be cyclic. In fact, if $K$ were cyclic, $G$ would involve operators of order $p^{m-1}$, which is contrary to the assumption made above.

As $K$ is non-cyclic it must involve more than one subgroup of order $p^{m-3}$, and the operators which are common to all of its subgroups of this order must constitute a characteristic subgroup with respect to which its quotient group is abelian and of type $(1,1,1, \cdots)$. Hence it results that $G$ must have an invariant subgroup of index $p^{4}$ which gives rise to a quotient group such that the operators of $K$ correspond to one of its non-cyclic subgroups of order $p^{2}$.

The operators of $K$ which correspond to operators of order $p$ in this quotient group cannot be powers of operators of higher order in $K$ and hence they must all be $p$ th powers of operators of $G$ which are not in $K$. That is, this quotient group of order $p^{4}$ must be such that each of its operators of order $p$ corresponding to $K$ is a power of an operator of order $p^{2}$ in the rest of 
this quotient group. This implies that this quotient group is abelian and of type $(2,2)$ when $p=2$, and when $p>2$ it must contain at least $p$ invariant cyclic subgroups of order $p^{2}$. As this is contrary to the fact that $G$ contains $p+1$ conjugate sets which involve generating operators of its maximal cyclic subgroups, we have proved that we arrive at an absurdity by assuming that $G$ does not involve any operator of order $p^{m-1}$, $m>3$.

When $p>2$ there are only two non-cyclic groups of order $p^{m}$ which involve operators of order $p^{m-1}, m>3$, and each of these clearly contains maximal cyclic subgroups of order $p^{a}$ which are transformed into themselves by more than $p^{a+1}$ operators of $G$. Hence it results that the three non-cyclic groups of order $2^{m}$ which were considered above in the second paragraph are the only non-cyclic groups of order $p^{m}$ in which every maximal cyclic subgroup is transformed into itself by at most $p$ times as many operators of the group as there are operators in this maximal subgroup. This completes the proof of the theorem in question, and hence we can assume that every non-cyclic group of order $p^{m}$, with the exception of the three of order $2^{m}$ which involve one and only one cyclic subgroup of order $2^{m-1}$, contains at least one maximal cyclic subgroup of order $p^{\alpha}$ which is transformed into itself by more than $p^{\alpha+1}$ operators of the group.

UNIVERSITY OF ILLINOIS.

\section{AN EXPRESSION FOR THE GENERAL TERM OF A RECURRING SERIES.}

BY PROFESSOR TSURUICHI HAYASHI.

Professor Arthur Ranum has given in the Bulletin, volume 17, No. 9, June, 1911, pages 457-461, an explicit form of the general term of a recurring series rationally in terms of the first few terms and the constants of the scale of relation. I will give here another more explicit and more convenient form without demonstration.

Let $u_{0}+u_{1}+u_{2}+\ldots+u_{n}+\ldots$ be any recurring series of order $n$, and let

$$
u_{m}=a_{1} u_{m-1}+a_{2} u_{m-2}+\cdots+a_{n} u_{m-n} \quad(m \geqq n)
$$

